TRANSACTIONS OF THE

AMERICAN MATHEMATICAL SOCIETY

Volume 354, Number 7, Pages 2759-2773

S 0002-9947(02)02974-

Article electronically published on March 14, 2002

\title{
VERTICES FOR CHARACTERS OF $p$-SOLVABLE GROUPS
}

\author{
GABRIEL NAVARRO
}

\begin{abstract}
Suppose that $G$ is a finite $p$-solvable group. We associate to every irreducible complex character $\chi \in \operatorname{Irr}(G)$ of $G$ a canonical pair $(Q, \delta)$, where $Q$ is a $p$-subgroup of $G$ and $\delta \in \operatorname{Irr}(Q)$, uniquely determined by $\chi$ up to $G$ conjugacy. This pair behaves as a Green vertex and partitions $\operatorname{Irr}(G)$ into "families" of characters. Using the pair $(Q, \delta)$, we give a canonical choice of a certain $p$-radical subgroup $R$ of $G$ and a character $\eta \in \operatorname{Irr}(R)$ associated to $\chi$ which was predicted by some conjecture of G. R. Robinson.
\end{abstract}

\section{INTRODUCTION}

Let $p$ be a prime and let $G$ be a finite $p$-solvable group. In this paper, we associate to every ordinary irreducible character $\chi \in \operatorname{Irr}(G)$ a canonical pair $(Q, \delta)$, where $Q$ is a $p$-subgroup of $G$ and $\delta \in \operatorname{Irr}(Q)$, which is uniquely determined by $\chi$ up to $G$-conjugacy. We say that $(Q, \delta)$ is a vertex of $\chi$, and we denote by

$$
\operatorname{Irr}(G \mid Q, \delta)
$$

the set of all $\chi \in \operatorname{Irr}(G)$ with vertex $(Q, \delta)$. (See Section 3 for this construction.)

If $G$ is a finite group and $S$ is a certain complete discrete valuation ring or a field of characteristic $p$, we know by work of J. A. Green that each indecomposable $S G$-module $V$ has an associated $G$-conjugacy class of $p$-subgroups $Q$ of $G$ called the vertices of $V$. Given an irreducible character $\chi \in \operatorname{Irr}(G)$, it is possible to find a (necessarily indecomposable) $S G$-module $V$ affording $\chi$, and therefore we might associate to $\chi$ a set of vertices in the Green sense. As is well-known, however, $\chi$ does not uniquely determine $V$, and different $S G$-modules affording $\chi$ can have non-isomorphic vertices. (Of course, this is not the case with the irreducible Brauer characters of $G$. Given $\varphi \in \operatorname{IBr}(G)$, we have that $\varphi$ uniquely determines up to isomorphism a simple $F G$-module $V$, where $F$ is an algebraically closed field of characteristic $p$, and therefore, irreducible Brauer characters do have vertices associated. If $Q$ is a $p$-subgroup of $G$, we denote by $\operatorname{IBr}(G \mid Q)$ the set of irreducible Brauer characters of $G$ with vertex $Q$.)

The pairs $(Q, \delta)$ that we associate to the irreducible character of the $p$-solvable groups behave, in certain aspects, as the Green vertices. In order to state their main properties, we remind the reader of some definitions and notation. First of all, the defect $d(\chi)$ of any character $\chi \in \operatorname{Irr}(G)$ is defined by the equation

$$
p^{d(\chi)} \chi(1)_{p}=|G|_{p} .
$$

Received by the editors March 10, 2001 and, in revised form, October 10, 2001.

2000 Mathematics Subject Classification. Primary 20C15.

Research partially supported by DGICYT and MEC. 
Recall that a $p$-subgroup $Q$ of $G$ is $p$-radical (in $G)$ if $Q=\mathbf{O}_{p}\left(\mathbf{N}_{G}(Q)\right.$ ). Also, every $p$-subgroup $Q$ of $G$ is contained in a $p$-radical subgroup of $G$ uniquely determined by $Q$ and $G$. This is called the radical closure of $Q$ in $G$ and is the last term $R$ of the chain $P_{i+1}=\mathbf{O}_{p}\left(\mathbf{N}_{G}\left(P_{i}\right)\right)$ starting with $Q=P_{0}$. If $Q$ is a $p$-subgroup of $G$ and $\delta \in \operatorname{Irr}(Q)$, we denote by $\mathbf{N}_{G}(Q, \delta)$ the elements of $\mathbf{N}_{G}(Q)$ which stabilize $\delta$.

Theorem A. Let $Q$ be a p-subgroup of a p-solvable group $G$ and let $\delta \in \operatorname{Irr}(Q)$.

(a) Suppose that $\delta=1_{Q}$ is the principal character of $Q$. Then restriction to p-regular elements defines a canonical bijection

$$
\operatorname{Irr}(G \mid Q, \delta) \rightarrow \operatorname{IBr}(G \mid Q) .
$$

(b) If $\chi \in \operatorname{Irr}(G)$ has vertex $(Q, \delta)$, then $d(\chi)=d(\delta)$.

(c) If $\chi \in \operatorname{Irr}(G)$ has vertex $(Q, \delta)$, then $\chi(g)=0$ whenever the $p$-part of $g$ lies in no $G$-conjugate of $Q$.

(d) If $\chi \in \operatorname{Irr}(G)$ has vertex $(Q, \delta)$, then

$$
\mathbf{O}_{p}\left(\mathbf{N}_{G}(Q, \delta)\right)=Q .
$$

(e) Suppose that $(Q, \delta)$ is a vertex of $\chi \in \operatorname{Irr}(G)$. Let $R$ be the radical closure of $Q$ in $G$. Then there is a defect group $D$ of the $p$-block of $\chi$ such that $Q \subseteq R \subseteq D$ and

$$
\mathbf{C}_{D}(Q)=\mathbf{Z}(Q) .
$$

(f) Suppose that $(Q, \delta)$ is a vertex of $\chi \in \operatorname{Irr}(G)$, and let $R$ be the radical closure of $Q$ in $G$. Then the induced character $\eta=\delta^{R}$ is irreducible.

(g) Suppose that $Q \triangleleft G$ and that $\delta$ is $G$-invariant. Then $\chi \in \operatorname{Irr}(G)$ has vertex $(Q, \delta)$ if and only if the restriction $\chi_{Q}$ contains $\delta$ and

$$
(\chi(1) / \delta(1))_{p}=|G: Q|_{p} .
$$

In the special case where $\delta=1_{Q}$, Theorem A recovers several well-known results on the irreducible Brauer characters of the $p$-solvable groups. Of course, Theorem A(a) gives a strong form of the Fong-Swan theorem that irreducible Brauer characters of $p$-solvable groups are liftable, or Theorem $\mathrm{A}(\mathrm{d})$ reproves that the vertices of the Brauer characters are $p$-radical (in $p$-solvable groups). Concerning part (e), the fact that vertices are "big" (that is, containing its own centralizer) in the defect groups is a celebrated general result of R. Knörr ([7]), although the part on the radical closure seems not have been noticed up to now.

When introducing a "new" object it is always pleasant to find applications, and we do have one for our pairs $(Q, \delta)$. A consequence drawn by G. R. Robinson of the well-known conjectures on representation theory of groups by E. C. Dade and himself is that, given $\chi \in \operatorname{Irr}(G)$, there always can be found a radical $p$-subgroup $R$ of $G$ which is "big" in a defect group of the $p$-block of $\chi$, and which has an irreducible character $\eta \in \operatorname{Irr}(R)$ with $d(\chi)=d(\eta)$. For $p$-solvable groups this is now a fact (see Theorem 2 of [11, or [1] for a partial result). Our Theorem A, parts (e) and (f), gives a canonical choice for Robinson's predicted $R$ and $\eta$ : if $(Q, \delta)$ is a vertex of $\chi$, it suffices to take $R$ the radical closure of $Q$ in $G$ and $\eta=\delta^{R}$.

Our aim when introducing the vertices $(Q, \delta)$ for the irreducible characters of the $p$-solvable groups was to explore the relationship between the characters of $G$ and the characters of certain local subgroups of $G$. In fact, our main concern is to find any connection between the sets $\operatorname{Irr}(G \mid Q, \delta)$ and $\operatorname{Irr}\left(\mathbf{N}_{G}(Q, \delta) \mid Q, \delta\right)$. For instance, 
by Theorem $\mathrm{A}(\mathrm{g})$, notice that when $\delta=1_{Q}$, the set $\operatorname{Irr}\left(\mathbf{N}_{G}(Q, \delta) \mid Q, \delta\right)$ consists of the Alperin weights. Hence, for $\delta=1_{Q}$, we have that

$$
|\operatorname{Irr}(G \mid Q, \delta)|=\left|\operatorname{Irr}\left(\mathbf{N}_{G}(Q, \delta) \mid Q, \delta\right)\right|
$$

(by Theorem A, (a) and (g), and the main result in [5]). This proves, of course, the Alperin weight conjecture for $p$-solvable groups.

Suppose now that $\chi \in \operatorname{Irr}(G)$ and let $(Q, \delta)$ be a vertex of $\chi$. By Theorem A(b), it is easy to check that a character $\chi \in \operatorname{Irr}(G)$ has $p^{\prime}$-degree if and only if $Q$ is a Sylow $p$-subgroup of $G$ and $\delta$ is linear. If this is the case, notice that $\operatorname{Irr}\left(\mathbf{N}_{G}(Q, \delta) \mid Q, \delta\right)$ consists exactly of those characters of $\mathbf{N}_{G}(Q, \delta)$ lying over $\delta$ (by Theorem $\mathrm{A}(\mathrm{g})$ ). Hence, by the Clifford correspondence, we have that $\left|\operatorname{Irr}\left(\mathbf{N}_{G}(Q, \delta) \mid Q, \delta\right)\right|$ is the number of irreducible characters of $\mathbf{N}_{G}(Q)$ lying over $\delta$. By using the main result of [6] it is possible to prove that whenever $Q \in \operatorname{Syl}_{p}(G)$ and $\delta \in \operatorname{Irr}(Q)$ is linear, again we have

$$
|\operatorname{Irr}(G \mid Q, \delta)|=\left|\operatorname{Irr}\left(\mathbf{N}_{G}(Q, \delta) \mid Q, \delta\right)\right|,
$$

proving a strong form of the McKay conjecture.

Our construction of the vertex pair $(Q, \delta)$ for $\chi \in \operatorname{Irr}(G)$ heavily uses the $p$ solvability of $G$. We do not know if it is possible to associate a similar canonical pair $(Q, \delta)$ to every irreducible character $\chi$ of every finite group $G$.

In order to prove some of the previous results, it is essential to establish the following property of the radical closure (which we find interesting on its own).

Theorem B. Suppose that $G$ is p-solvable and let $N \triangleleft G$. Suppose that $\theta \in \operatorname{IBr}(N)$ has $p^{\prime}$-degree. Let $Q$ be any p-subgroup of $G$ containing a Sylow p-subgroup of $N$, and let $R$ be its radical closure in $G$. If $\theta$ is $Q$-invariant, then $\theta$ is $R$-invariant.

I would like to thank M. Isaacs for many useful conversations on this subject.

\section{INDUCING CHARACTERS}

In this section, instead of restricting ourselves to a single prime $p$, we work with a set of primes $\pi$ with the same amount of work. Our goal now is to associate to every irreducible $\chi \in \operatorname{Irr}(G)$ of a $\pi$-separable group $G$ a unique (up to $G$-conjugacy) pair $(W, \gamma)$, where $W \subseteq G$ and $\gamma \in \operatorname{Irr}(W)$, satisfying certain properties. We will obtain $(W, \gamma)$ by repeated Clifford induction from the "right" normal subgroups. We remind the reader that a group $G$ is $\pi$-separable if it has a normal series $1=$ $G_{k}<\cdots<G_{0}=G$ such that the factors $G_{i} / G_{i+1}$ are either $\pi$ - or $\pi^{\prime}$-groups. Of course, the $p$-solvable groups are the $p$-separable groups. Also, $\pi$-separable groups have Hall $\pi$-subgroups, and any two of them are $G$-conjugate.

To find the pairs $(W, \gamma)$, we use $\pi$-special characters, and next we remind the reader of their definition and main properties. If $G$ is $\pi$-separable, an irreducible $\chi \in \operatorname{Irr}(G)$ is said to be $\pi$-special if $\chi(1)$ is a $\pi$-number and for every subnormal subgroup $N \triangleleft \triangleleft G$ and $\theta \in \operatorname{Irr}(N)$ under $\chi$, the determinantal order of $\theta$ is a $\pi$ number. (Recall that the determinantal order of $\theta$ is the order of the linear character $\operatorname{det} \mathcal{X}$, where $\mathcal{X}$ is any representation affording $\theta$.) Of course, if $G$ is a $\pi$-group, then all irreducible characters of $G$ are $\pi$-special. Also, by the definition, normal irreducible constituents of $\pi$-special characters are again $\pi$-special.

Let us write down for the reader's convenience the properties of the $\pi$-special characters that we are going to use later on. If $p$ is a prime and $\chi \in \operatorname{Irr}(G)$, we denote by $\chi^{0}$ the restriction of $\chi$ to the set $G^{0}$ of $p$-regular elements of $G$. 
(2.1) Theorem. Suppose that $G$ is $\pi$-separable, let $H$ be a Hall $\pi$-subgroup of $G$, and let $\mathcal{X}_{\pi}(G)$ be the set of irreducible $\pi$-special characters of $G$.

(a) If $N \triangleleft G$ is a $\pi^{\prime}$-group and $\chi \in \mathcal{X}_{\pi}(G)$, then $N \subseteq \operatorname{ker}(\chi)$.

(b) If $G / M$ is a $\pi^{\prime}$-group, then restriction defines a bijection between $\mathcal{X}_{\pi}(G)$ and the set of $\pi$-special $G$-invariant characters of $M$.

(c) If $J \subseteq G$ has $\pi^{\prime}$-index, then restriction defines a one-to-one map between $\mathcal{X}_{\pi}(G)$ and $\mathcal{X}_{\pi}(J)$.

(d) If $K$ is a $\pi$-complement of $G$, then

$$
\left|\mathcal{X}_{\pi}(G)\right|=\left|\operatorname{Irr}\left(\mathbf{N}_{G}(K) / K\right)\right| .
$$

(e) If $G$ is p-solvable and $\operatorname{IBr}_{p^{\prime}}(G)$ is the set of irreducible p-Brauer characters of $G$ of $p^{\prime}$-degree, then restriction to p-regular elements defines a bijection

$$
\mathcal{X}_{p^{\prime}}(G) \rightarrow \operatorname{IBr}_{p^{\prime}}(G) .
$$

(f) Suppose that $G$ is p-solvable and let $N \triangleleft G$. Suppose that $\chi \in \operatorname{Irr}(G)$ and $\theta \in \operatorname{Irr}(N)$ are $p^{\prime}$-specials. Then $\chi^{0} \in \operatorname{IBr}(G)$ lies over $\theta^{0} \in \operatorname{IBr}(N)$ iff $\chi$ lies over $\theta$.

Proof. Part (a) follows from Corollary (4.2) of [2. Part (b) is Proposition (4.3) of [2. Part (c) follows from Proposition (6.1) of [2]. Part (d) is deeper and can be found as Corollary (1.16) of [12]. Part (e) easily follows from Lemma (5.4) and Corollary (10.3) of 3]. Now, we prove part (f). If $\theta$ lies over $\chi$, it is clear that $\chi^{0}$ lies under $\theta^{0}$. Conversely, suppose that $\chi^{0}$ lies over $\theta^{0}$. Let $H$ be a $p$-complement of $G$. Then $H \subseteq G^{0}$, and we have that $\chi_{H} \in \operatorname{Irr}(H)$ lies over $\theta_{H \cap N} \in \operatorname{Irr}(N \cap H)$. Therefore, some irreducible constituent $\eta$ of $\chi_{N}$ must lie over $\theta_{N \cap H}$. However, $\eta$ is $p^{\prime}$-special. By part (c), we have that $\eta_{N \cap H}=\theta_{N \cap H}$, and therefore $\eta=\theta$, as desired.

Another remarkable property of special characters is that if $\alpha \in \operatorname{Irr}(G)$ is $\pi$ special and $\beta \in \operatorname{Irr}(G)$ is $\pi^{\prime}$-special, then $\alpha \beta \in \operatorname{Irr}(G)$ (Proposition (7.1) of [2]). In fact, this factorization is unique. The irreducible characters of $G$ of the form $\alpha \beta$ are called $\pi$-factorable. Sometimes if $\chi \in \operatorname{Irr}(G)$ is $\pi$-factorable, we write

$$
\chi=\chi_{\pi} \chi_{\pi^{\prime}},
$$

where $\chi_{\pi}$ is $\pi$-special and $\chi_{\pi^{\prime}}$ is $\pi^{\prime}$-special. Since normal irreducible constituents of $\pi$-specials are $\pi$-special, the same happens with $\pi$-factorable characters.

(2.2) Theorem. Suppose that $G$ is $\pi$-separable, and let $N, M \triangleleft G$. If the irreducible constituents of $\chi_{N}$ and of $\chi_{M}$ are $\pi$-factorable, then the irreducible constituents of $\chi_{N M}$ are also $\pi$-factorable.

Proof. We argue by induction, first on $|G|$, and then on $|G: N|+|G: M|$. If $\delta$ is an irreducible constituent of $\chi_{N M}$, we have that the irreducible constituents of $\delta_{M}$ and of $\delta_{N}$ are $\pi$-factorable. So by induction, we may assume that $G=N M$, and we should prove that $\chi$ is $\pi$-factorable. Now suppose that $N \subseteq K \triangleleft G$ is a proper normal subgroup of $G$, and let $\epsilon$ lie under $\chi$. Then the irreducible constituents of $\epsilon_{N}$ are irreducible constituents of $\chi_{N}$, and therefore they are $\pi$-factorable. On the other hand, the irreducible constituents of $\epsilon_{K \cap M}$ lie under irreducible constituents of $\chi_{M}$, and therefore, they are also $\pi$-factorable. Arguing by induction, we obtain that $\epsilon$ is $\pi$-factorable, and again by induction, that $\chi$ is $\pi$-factorable. So we may assume that $N$ and $M$ are maximal normal subgroups of $G$. Hence, each $G / N$ and $G / M$ is a $\pi$-group or a $\pi^{\prime}$-group. Now, let $\theta \in \operatorname{Irr}(N)$ be under $\chi$, and let 
$\eta \in \operatorname{Irr}(N \cap M)$ be under $\theta$. Hence $\eta$ lies under $\chi$, and therefore there is some $\varphi \in \operatorname{Irr}(M)$ over $\eta$ and under $\chi$. The theorem now follows from Corollary (2.8) of [3].

A pair $(G, \chi)$ is just a group $G$ with an irreducible character $\chi \in \operatorname{Irr}(G)$. Also, if $H \subseteq G, \alpha \in \operatorname{Irr}(H)$ and $g \in G$, then $(H, \alpha)^{g}=\left(H^{g}, \alpha^{g}\right)$, where $\alpha^{g} \in \operatorname{Irr}\left(H^{g}\right)$ is the character satisfying

$$
\alpha^{g}\left(h^{g}\right)=\alpha(h)
$$

for $h \in H$.

(2.3) Corollary. Suppose that $G$ is $\pi$-separable and let $\chi \in \operatorname{Irr}(G)$. Then there is, up to $G$-conjugacy, a unique pair $(N, \theta)$ which is maximal subject to $N$ being a normal subgroup of $G$ and $\theta$ being a $\pi$-factorable irreducible character of $N$ lying under $\chi$.

Proof. If $(M, \varphi)$ is another $\pi$-factorable maximal pair under $(G, \chi)$, then the irreducible constituents of $\chi_{N M}$ are $\pi$-factorable by Theorem (2.2). By maximality, we have that $N=M$ and therefore, by Clifford's theorem, $(N, \theta)^{g}=(M, \varphi)$.

(2.4) Corollary. Suppose that $G$ is $\pi$-separable, and let $\chi \in \operatorname{Irr}(G)$. Let $(N, \theta)$ be a maximal normal $\pi$-factorable pair under $\chi$. If $\theta$ is $G$-invariant, then $G=N$ and $\chi=\theta$.

Proof. Suppose that $N$ is proper in $G$. Let $M / N$ be a chief factor of $G$. Also, let $\eta \in \operatorname{Irr}(M)$ lie under $\chi$ and over $\theta$. Now, by the uniqueness of the decomposition of a character as a $\pi$-special times a $\pi^{\prime}$-special, it follows that the $\pi$-part and the $\pi^{\prime}$-part of $\theta$ are $G$-invariant. Since $M / N$ is a $\pi$-group or a $\pi^{\prime}$-group, it follows by Proposition (2.7) of [3] that $\eta$ is $\pi$-factorable. This contradicts the maximality of $(N, \theta)$.

Given a $\pi$-separable group $G$ and $\chi \in \operatorname{Irr}(G)$, we define a canonical (up to $G$ conjugacy) pair $(W, \gamma)$ satisfying $W \subseteq G, \gamma \in \operatorname{Irr}(W)$ is $\pi$-factorable, and $\gamma^{G}=\chi$. We do this inductively on $|G|$. If $\chi$ is $\pi$-factorable, then we let $(W, \gamma)=(G, \chi)$. If $\chi$ is not $\pi$-factorable, then let $(N, \theta)$ be a maximal $\pi$-factorable normal pair under $\chi$. Let $T=I_{G}(\theta)$ be the stabilizer of $\theta$ in $G$ and let $\psi \in \operatorname{Irr}(T \mid \theta)$ be the Clifford correspondent of $\chi$ over $\theta$. By Corollary (2.4), we have that $T$ is proper in $G$. Then, by induction, we have defined a pair $(W, \gamma)$ for $(T, \psi)$. We call every $G$-conjugate of every pair $(W, \gamma)$ arising this way a nucleus via normal pairs for $\chi$. By definition and Theorem $(2.3)$, notice that if $(W, \gamma)$ and $(U, \eta)$ are nuclei for $\chi$, then there is $g \in G$ such that

$$
(W, \gamma)^{g}=(U, \eta)
$$

(The analogous construction of the nucleus of a character via subnormal pairs is done in [3. Our normal construction is, however, essential for our purposes here.) Since there will be no other nuclei in this paper, we will simply refer to $(W, \gamma)$ as a nucleus for $\chi$.

\section{VERTICES FOR CHARACTERS}

Suppose now that $G$ is $p$-solvable, and let $\chi \in \operatorname{Irr}(G)$. Let $Q$ be a $p$-subgroup of $G$ and let $\delta \in \operatorname{Irr}(Q)$. We say that $(Q, \delta)$ is a vertex of $\chi$ if there is a nucleus $(W, \gamma)$ for $\chi$ such that $Q \in \operatorname{Syl}_{p}(W)$ and $\beta_{Q}=\delta$, where $\beta=\gamma_{p}$ is the $p$-part of $\gamma$. 
Suppose now that $(E, \eta)$ is another vertex of $\chi$. We claim that there is $x \in G$ such that

$$
(Q, \delta)^{x}=(E, \eta) .
$$

We know that there is a $g \in G$ such that $E$ is a Sylow $p$-subgroup of $W^{g}$ and $\eta=\left(\left(\gamma^{g}\right)_{p}\right)_{E}$. Now, $Q^{g}$ is a Sylow $p$-subgroup of $W^{g}$, and therefore $Q^{g}=E^{w^{g}}$ for some $w \in W$. Hence $Q^{w^{-1} g}=E$. Also,

$$
\begin{aligned}
\delta^{w^{-1} g} & =\left(\left(\gamma_{p}\right)_{Q}\right)^{w^{-1} g}=\left(\left(\gamma_{p}\right)^{w^{-1} g}\right)_{Q^{w^{-1} g}} \\
& =\left(\left(\gamma_{p}\right)^{w^{-1} g}\right)_{E}=\left(\left(\gamma_{p}\right)^{g}\right)_{E}=\left(\left(\gamma^{g}\right)_{p}\right)_{E}=\eta,
\end{aligned}
$$

as desired.

We denote by $\operatorname{Irr}(G \mid Q, \delta)$ the set of irreducible characters $\chi$ with vertex $(Q, \delta)$.

The following easily follows from the definition of the defect of a character.

(3.1) Lemma. Suppose that $\psi^{G}=\chi \in \operatorname{Irr}(G)$. Then $d(\psi)=d(\chi)$.

Proof. Suppose that $\psi \in \operatorname{Irr}(H)$. Then

$$
p^{d(\chi)}=|G|_{p} / \chi(1)_{p}=|G|_{p} /|G: H|_{p} \psi(1)_{p}=|H|_{p} / \psi(1)_{p}=p^{d(\psi)},
$$

as desired.

Next is Theorem A(b).

(3.2) Theorem. Let $G$ be $p$-solvable and suppose that $\chi \in \operatorname{Irr}(G)$ has vertex $(Q, \delta)$. Then $d(\chi)=d(\delta)$.

Proof. Let $(W, \gamma)$ be a nucleus for $\chi$ such that $Q \in \operatorname{Syl}_{p}(W)$ and $\left(\gamma_{p}\right)_{Q}=\delta$. Hence by Lemma (3.1) we have

$$
p^{d(\chi)}=p^{d(\gamma)}=p^{d\left(\gamma_{p}\right)}=|W|_{p} / \gamma_{p}(1)=|Q| / \delta(1)=p^{d(\delta)},
$$

as desired.

Next is Theorem A(c).

(3.3) Theorem. Let $G$ be p-solvable and suppose that $\chi \in \operatorname{Irr}(G)$ has vertex $(Q, \delta)$. Let $g \in G$. If no $G$-conjugate of $g_{p}$ lies in $Q$, then $\chi(g)=0$.

Proof. Let $(W, \gamma)$ be a nucleus for $\chi$ such that $Q \in \operatorname{Syl}_{p}(W)$ and $\left(\gamma_{p}\right)_{Q}=\delta$. By hypothesis, no $G$-conjugate of $g$ lies in $W$. Since $\chi=\gamma^{G}$, we have $\chi(g)=0$, by the induction formula.

In order to understand a bit more about the vertices $(Q, \delta)$, we proceed to prove Theorem $\mathrm{A}(\mathrm{g})$. For that we need a few easy lemmas.

(3.4) Lemma. Let $N \triangleleft G$, let $\theta \in \operatorname{Irr}(N)$ and let $\chi \in \operatorname{Irr}(G \mid \theta)$. Suppose that $d(\chi)=d(\theta)$. If $N \subseteq M \triangleleft G$ and $\eta \in \operatorname{Irr}(M)$ lies under $\chi$, then $d(\eta)=d(\chi)$.

Proof. Since $\eta$ lies over some $G$-conjugate of $\theta$, it is no loss to assume that $\eta$ lies over $\theta$. Now, $\chi(1)_{p} / \eta(1)_{p}$ divides $|G: M|_{p}$ and $\eta(1)_{p} / \theta(1)_{p}$ divides $|M: N|_{p}$. Since

$$
\left(\chi(1)_{p} / \eta(1)_{p}\right)\left(\eta(1)_{p} / \theta(1)_{p}\right)=\chi(1)_{p} / \theta(1)_{p}=|G: N|_{p}=|G: M|_{p}|M: N|_{p},
$$

the proof of the lemma easily follows. 
(3.5) Lemma. Let $M \triangleleft G$. Suppose that $\gamma=\alpha \beta \in \operatorname{Irr}(M)$, where $\alpha$ is $p^{\prime}$-special, and $\beta$ is p-special and invariant under some Sylow p-subgroup $P$ of $G$. Suppose that $\chi \in \operatorname{Irr}(G)$ lies over $\gamma$ with $d(\chi)=d(\gamma)$. Then there is a nucleus $(W, \rho)$ of $\chi$ where $W / M$ is a $p^{\prime}$-group and $\left(\rho_{p}\right)_{M}=\beta$. In particular, if $Q \in \operatorname{Syl}_{p}(M)$, then $\left(Q, \beta_{Q}\right)$ is a vertex for $\chi$.

Proof. We argue by induction on $|G|$. Let $(N, \theta)$ be a maximal normal pair over $(M, \gamma)$. Also, let $Q=P \cap N \in \operatorname{Syl}_{p}(N)$. We have that $\beta$ is $Q$-invariant. Therefore, so is $\beta_{Q \cap M}$. By Lemma (3.4), $d(\gamma)=d(\theta)$. This implies that

$$
\theta_{p}(1) / \beta(1)=|N: M|_{p}=|Q: Q \cap M| .
$$

Now, $\theta_{p}$ lies over $\beta$, and therefore $\left(\theta_{p}\right)_{Q} \in \operatorname{Irr}(Q)$ lies over $\beta_{Q \cap M}$. By degrees, we see that $\beta_{Q \cap M}$ induces $\left(\theta_{p}\right)_{Q}$. Since $\beta_{Q \cap M}$ is $Q$-invariant, this implies that $Q \cap M=Q$, by Problem (6.1) of [4], for instance. Hence we have that $N / M$ is a $p^{\prime}$-group.

Now, let $\psi$ be the Clifford correspondent of $\chi$ over $\theta$. By Lemma (3.1), we have $d(\psi)=d(\gamma)$. If $T<G$, by induction, we are done. If $T=G$, by Corollary (2.4), $N=G$, and in this case the proof of the lemma is clear.

The following is a restatement of Theorem $\mathrm{A}(\mathrm{g})$.

(3.6) Theorem. Let $G$ be a p-solvable group, and fix a pair $(Q, \delta)$ with $d(\delta)=d$. Then the set $\operatorname{Irr}\left(\mathbf{N}_{G}(Q, \delta) \mid Q, \delta\right)$ consists exactly of those $\chi \in \operatorname{Irr}\left(\mathbf{N}_{G}(Q, \delta)\right)$ lying over $\delta$ such that $d(\chi)=d=d(\delta)$.

Proof. We may assume that $\mathbf{N}_{G}(Q, \delta)=G$. So we have that $Q \triangleleft G$ and $\delta$ is $G$-invariant. Let $\chi \in \operatorname{Irr}(G \mid Q, \delta)$. Then there is a nucleus $(W, \gamma)$ such that $Q \in$ $\operatorname{Syl}_{p}(W)$ and $\left(\gamma_{p}\right)_{Q}=\delta$. Since $Q \triangleleft G$, we have that $Q \subseteq \operatorname{ker}\left(\gamma_{p^{\prime}}\right)$, and therefore $\gamma$ lies over $\delta$. Hence $\chi$ lies over $\delta$. By Theorem (3.2), we know that $d(\chi)=d$.

Conversely, suppose that $\chi \in \operatorname{Irr}(G \mid \delta)$ has defect $d$. By Lemma (3.5), we deduce that $(Q, \delta)$ is a vertex of $\chi$, and the theorem is proven.

\section{Characters and Radical subgroups}

This is one of our key results.

(4.1) Theorem. Suppose that $G$ is p-solvable and let $N \triangleleft G$. Suppose that $\theta \in$ $\mathcal{X}_{p^{\prime}}(N)$ is $Q$-invariant, where $Q$ is a p-subgroup of $G$ containing a Sylow p-subgroup of $N$. Suppose that $Q \subseteq R$ is a p-subgroup of $G$ such that $\mathbf{N}_{N}(Q) \subseteq \mathbf{N}_{N}(R)$. Then $\theta$ is R-invariant.

Proof. We argue by induction on $|R: Q|$. If $Q=R$, there is nothing to prove. So we assume that $Q<R$. Since $Q \subseteq R \subseteq N R, N \triangleleft N R$ and $\mathbf{N}_{N}(Q) \subseteq \mathbf{N}_{N}(R)$, it is no loss to assume that $N R=G$.

Write $D=Q \cap N \in \operatorname{Syl}_{p}(N)$. We have that $D \triangleleft \mathbf{N}_{N}(Q)$. Let $E=Q^{R} \triangleleft R$. Since $\mathbf{N}_{N}(Q)$ normalizes $Q$ and $R$, it follows that $\mathbf{N}_{N}(Q)$ normalizes $E$. Since $E<R$, we have that $|E: Q|<|R: Q|$, and by induction, we conclude that $\theta$ is $E$-invariant. We claim that

$$
\mathbf{N}_{N}(E)=\mathbf{N}_{N}(Q)
$$

We already have seen that $\mathbf{N}_{N}(Q) \subseteq \mathbf{N}_{N}(E)$. Now, since $D=E \cap N$, we have that $\mathbf{N}_{G}(E) \subseteq \mathbf{N}_{G}(D)$. Hence $D \triangleleft \mathbf{N}_{G}(E)$. Now, $D=N \cap Q \subseteq \mathbf{N}_{N}(Q) \subseteq \mathbf{N}_{N}(E) \subseteq N$, and therefore $\mathbf{N}_{N}(E) / D$ is a normal $p^{\prime}$-subgroup of $\mathbf{N}_{G}(E) / D$. Since $E / D$ is a 
normal $p$-subgroup of $\mathbf{N}_{G}(E) / D$, we conclude that $\left[\mathbf{N}_{N}(E), E\right] \subseteq D \subseteq Q$. Hence $\left[\mathbf{N}_{N}(E), Q\right] \subseteq Q$, and we deduce that $\mathbf{N}_{N}(E) \subseteq \mathbf{N}_{N}(Q)$. This proves the claim.

Now, we have that $\mathbf{N}_{N}(E)=\mathbf{N}_{N}(Q) \subseteq \mathbf{N}_{N}(R)$. If $|R: E|<|R: Q|$, since $\theta$ is $E$-invariant, by induction we will have that $\theta$ is $R$-invariant, and the theorem would follow in this case. So we may assume that $Q \triangleleft R$.

Now, let $M=N Q \triangleleft N R=G$. Notice that $Q \in \operatorname{Syl}_{p}(M)$ and that $R \cap M=Q$. Thus $R \in \operatorname{Syl}_{p}(G)$. We claim that

$$
\mathbf{N}_{G}(R)=\mathbf{N}_{G}(Q) \text {. }
$$

Since $R \cap M=Q$, we have that $\mathbf{N}_{G}(R) \subseteq \mathbf{N}_{G}(Q)$. Hence, $\mathbf{N}_{N}(R) \subseteq \mathbf{N}_{N}(Q)$, and by hypothesis we have that $\mathbf{N}_{N}(R)=\mathbf{N}_{N}(Q)$. Now, since $G=N R$, then $\mathbf{N}_{G}(R)=R \mathbf{N}_{N}(R)=R \mathbf{N}_{N}(Q)$. Also, since $Q \triangleleft R$, we have that $R \subseteq \mathbf{N}_{G}(Q)$ and again $\mathbf{N}_{G}(Q)=R \mathbf{N}_{N}(Q)=\mathbf{N}_{G}(R)$, as claimed.

Now, we have

$$
\mathbf{N}_{G}(R) / R \cong \mathbf{N}_{M}(Q) / Q
$$

By Theorem (2.1.d), we have

$$
\left|\mathcal{X}_{p^{\prime}}(G)\right|=\left|\operatorname{Irr}\left(\mathbf{N}_{G}(R) / R\right)\right|=\left|\operatorname{Irr}\left(\mathbf{N}_{M}(Q) / Q\right)\right|=\left|\mathcal{X}_{p^{\prime}}(M)\right| .
$$

Now, by Theorem (2.1.b), we have

$$
\left|\mathcal{X}_{p^{\prime}}(G)\right|=\left|\mathcal{X}_{p^{\prime}, R}(M)\right|,
$$

where $\mathcal{X}_{p^{\prime}, R}(M)$ is the set of $p^{\prime}$-special characters of $M$ which are $R$-invariant. Hence, we conclude that all $p^{\prime}$-special characters of $M$ are $R$-invariant. Now, since $\theta$ is $Q$-invariant, it follows that $\theta$ is $M$-invariant. Since $N \triangleleft M$ and $M / N$ is a $p$-group, it follows by Theorem (2.1.b) that there exists $\eta \in \mathcal{X}_{p^{\prime}}(M)$ extending $\theta$. Since $\eta$ is $p^{\prime}$-special, we conclude that $\eta$ is $R$-invariant. Hence, $\theta$ is $R$-invariant, as desired.

Next is Theorem A(d) of the Introduction.

(4.2) Theorem. Let $G$ be $p$-solvable and suppose that $(Q, \delta)$ is a vertex of $\chi \in$ $\operatorname{Irr}(G)$. Then

$$
\mathbf{O}_{p}\left(\mathbf{N}_{G}(Q, \delta)\right)=Q .
$$

Proof. We argue by induction on $|G|$. If $\chi$ is $p$-factorable, then $(G, \chi)$ is a nucleus of $\chi, Q \in \operatorname{Syl}_{p}(G)$, and therefore $\mathbf{O}_{p}\left(\mathbf{N}_{G}(Q, \delta)\right)=Q$, in this case.

Now, we may find a nucleus $(W, \gamma)$ for $\chi$ and a normal $p$-factorable pair $(N, \theta)$ such that if $T=I_{G}(\theta)$, we have that $N \subseteq W \subseteq T, Q \in \operatorname{Syl}_{p}(W)$, $\gamma$ lies over $\theta$, $\left(\gamma_{p}\right)_{Q}=\delta$ and $(W, \gamma)$ is a nucleus for $\psi=\gamma^{T}$ (the Clifford correspondent of $\chi$ over $\theta)$. Also, notice that $Q \cap N \in \operatorname{Syl}_{p}(N), Q \in \operatorname{Syl}_{p}(Q N)$, and $\left(\gamma_{p}\right)_{N Q}$ is the unique $p$-special extension of $\delta$ to $N Q$ (by Theorem (2.1.c)). Since $\psi \in \operatorname{Irr}(T \mid Q, \delta)$, by induction, we have that

$$
\mathbf{O}_{p}\left(\mathbf{N}_{T}(Q, \delta)\right)=Q .
$$

Let $R=\mathbf{O}_{p}\left(\mathbf{N}_{G}(Q, \delta)\right)$, so that $Q \subseteq R$.

Now, notice that $\mathbf{N}_{N}(Q, \delta)=\mathbf{N}_{N}(Q)$. This is because $\delta$ extends to $N Q$, and then every element in $N Q$ normalizing $Q$ fixes $\delta$. Now, $\mathbf{N}_{N}(Q) \subseteq \mathbf{N}_{G}(Q, \delta)$, and since $R \triangleleft \mathbf{N}_{G}(Q, \delta)$, we have that $\mathbf{N}_{N}(Q) \subseteq \mathbf{N}_{N}(R)$. By Theorem (4.1), $\theta_{p^{\prime}}$ is $R$-invariant. Now, if $\beta=\gamma_{p}$, we already know that $\beta_{N Q}$ is the unique $p$-special extension of $\delta$ to $N Q$, and that $\beta_{N}=e \theta_{p}$ for some integer $p$. If $r \in R$, we have 
that $r$ normalizes $Q$ and fixes $\delta$. Therefore, $r$ normalizes $N Q$. Then $\left(\beta_{N Q}\right)^{r}$ is another $p$-special extension of $\delta$ to $N Q$. By uniqueness, $\beta_{N Q}$ is $r$-invariant. Since $\left(\beta_{N Q}\right)_{N}=e \theta_{p}$, we deduce that $\theta_{p}$ is $r$-invariant. Hence, we have seen that $R$ fixes $\theta_{p}$ and $\theta_{p^{\prime}}$. Therefore $r \in T$, and we conclude that $R \subseteq T$. In particular, $R \triangleleft \mathbf{N}_{T}(Q, \delta)$. Since $Q=\mathbf{O}_{p}\left(\mathbf{N}_{T}(Q, \delta)\right)$, we deduce that $Q=R$, as desired.

Now, we draw some consequences of Theorem (4.1). We remind the reader that if $Q$ is any $p$-subgroup of $G$, the radical closure of $Q$ in $G$ is the last term $R$ of the chain $P_{i+1}=\mathbf{O}_{p}\left(\mathbf{N}_{G}\left(P_{i}\right)\right)$ starting with $Q=P_{0}$. Since the radical closure of $Q$ in $G$ is uniquely determined by $Q$ and $G$, it follows that $\mathbf{N}_{G}(Q) \subseteq \mathbf{N}_{G}(R)$.

(4.3) Corollary. Suppose that $G$ is p-solvable and let $N \triangleleft G$. Suppose that $\theta \in$ $\mathcal{X}_{p^{\prime}}(N)$ is $Q$-invariant, where $Q$ is a p-subgroup of $G$ containing a Sylow p-subgroup of $N$. If $R$ is the p-radical closure of $Q$ in $G$, then $\theta$ is $R$-invariant.

Proof. We have that $Q \subseteq R$ and $\mathbf{N}_{G}(Q) \subseteq \mathbf{N}_{G}(R)$. Hence, $\mathbf{N}_{N}(Q) \subseteq \mathbf{N}_{N}(R)$, and Theorem (4.1) applies.

Next is Theorem B of the introduction.

(4.4) Corollary. Suppose that $G$ is p-solvable and let $N \triangleleft G$. Suppose that $\theta \in$ $\operatorname{IBr}(N)$ has $p^{\prime}$-degree and is $Q$-invariant, where $Q$ is a p-subgroup of $G$ containing a Sylow p-subgroup of $N$. If $R$ is the p-radical closure of $Q$ in $G$, then $\theta$ is $R$ invariant.

Proof. By Theorem (2.1.e), we can find a unique $\eta \in \mathcal{X}_{p^{\prime}}(N)$ such that $\eta^{0}=\theta$, where $\eta^{0}$ is the restriction of $\eta$ to the $p$-regular elements of $N$. By uniqueness in Theorem (2.1.e), we have that $\eta$ is $Q$-invariant. Hence, by Corollary (4.3), we have that $\eta$ is $R$-invariant. Hence, $\theta=\eta^{0}$ is also $R$-invariant.

We find the following result a little surprising.

(4.5) Theorem. Suppose that $G$ is p-solvable and let $\chi \in \operatorname{Irr}(G)$. Suppose that $(N, \theta)$ is a normal maximal $p$-factorable pair under $\chi$. If $Q \in \operatorname{Syl}_{p}(N)$, then $Q=$ $\mathbf{O}_{p}\left(\mathbf{N}_{G}(Q)\right)$.

Proof. Write $\theta=\theta_{p} \theta_{p^{\prime}}$, where $\theta_{p}$ is $p$-special and $\theta_{p^{\prime}}$ is $p^{\prime}$-special. Let $R=$ $\mathbf{O}_{p}\left(\mathbf{N}_{G}(Q)\right)$. By Corollary (4.3), we know that $\theta_{p^{\prime}}$ is $R$-invariant. By the Frattini argument, we have that $G=N \mathbf{N}_{G}(Q)$. Then we have that $\mathbf{N}_{N}(Q) / Q$ is a $p^{\prime}$-subgroup of $\mathbf{N}_{G}(Q) / Q$. Since $R / Q$ is a $p$-subgroup of $\mathbf{N}_{G}(Q) / Q$, we conclude that $R \cap \mathbf{N}_{N}(Q)=Q$. Also, we have that $R N \triangleleft G$. Now, $R N / N$ is a $p$-group, and by Proposition (2.7) of [3], all irreducible constituents of $\theta^{R N}$ are $p$-factorable. By maximality, we conclude that $N R=N$, and thus $R=Q$, as required.

(4.6) Corollary. Suppose that $G$ is p-solvable and let $\chi \in \operatorname{Irr}(G)$ with vertex $(Q, \delta)$. Then there is $N \triangleleft G$ such that $Q \cap N$ is p-radical in $G$.

Proof. Let $(W, \gamma)$ be a nucleus for $\chi$ such that $Q \in \operatorname{Syl}_{p}(W)$ and $(W, \gamma)$ lies over $(N, \theta)$, a normal maximal factorable pair under $\chi$. Then $Q \cap N \in \operatorname{Syl}_{p}(N)$, and the result follows from Theorem (4.5).

From Corollary (4.6), it easily follows that vertices contain $\mathbf{O}_{p}(G)$, although this is immediate from their definition.

Suppose that $G$ is $p$-solvable, let $\chi \in \operatorname{Irr}(G)$ and let $(Q, \delta)$ be a vertex of $\chi$. In view of Theorems (4.1) and (4.5), it is natural to ask if $Q$ needs to be $p$-radical in $G$. The answer is no. 
(4.7) Example. Set $p=2$. Consider $H=N P$, where $N \triangleleft H$ is cyclic of order 5 and $P$ is cyclic of order 4 . For instance, suppose that $N=\langle x\rangle$ and $P=\langle y\rangle$, where $x=(12345)$ and $y=(2354)$. Hence $x^{y}=x^{2}$ and $H \subseteq S_{5}$. Also, $y^{2}=(25)(34)$. Now, take $H$ acting on $V=C_{2} \times C_{2} \times C_{2} \times C_{2} \times C_{2}$, and let $G=V H$ be the semidirect product. Now, let $\theta=1 \times \lambda \times 1 \times 1 \times \lambda \in \operatorname{Irr}(V)$, where $\lambda \neq 1$. Hence, the inertia group of $\theta$ in $G$ is $T=V\left\langle y^{2}\right\rangle$. If $\mu \in \operatorname{Irr}(T)$ lies over $\theta$, then $\mu^{G}=\chi \in \operatorname{Irr}(G)$, $(T, \mu)$ is a normal nucleus of $\chi$, and $T$ is not $p$-radical.

\section{Proof of Theorem A (e) And (f)}

We start with the following result.

(5.1) Theorem. Let $\chi \in \operatorname{Irr}(G)$ and let $(W, \gamma)$ be a nucleus for $\chi$. Let $Q \in$ $\operatorname{Syl}_{p}(W)$ and assume that $Q \subseteq R$ is a p-subgroup of $G$ such that $\mathbf{N}_{W}(Q) \subseteq \mathbf{N}_{W}(R)$. Write $\gamma=\alpha \beta$, where $\alpha$ is $p^{\prime}$-special and $\beta$ is p-special. Then $\left(\beta_{Q}\right)^{R} \in \operatorname{Irr}(R)$.

Proof. We argue by induction on $|G|$. Write $\delta=\beta_{Q}$. By Theorem (2.1.c), we know that $\delta \in \operatorname{Irr}(Q)$. Let $(N, \theta)$ be a maximal $p$-factorable pair such that $(W, \gamma)$ is a nucleus for $\psi$, the Clifford correspondent of $\chi$ over $\theta$, and $\gamma$ lies over $\theta$. Write $T=I_{G}(\theta)$. Also, write $\theta=\theta_{p} \theta_{p^{\prime}}$, where $\theta_{p}$ is $p$-special and $\theta_{p^{\prime}}$ is $p^{\prime}$-special. In particular, we have that $\theta_{p^{\prime}}$ is $Q$-invariant. If $T=G$, then by Corollary (2.4), we have that $W=G$. Hence, $Q=R$, and in this case we already know that $\delta$ is irreducible. So we may assume that $T<G$.

We claim that $\mathbf{N}_{W}(Q) \subseteq \mathbf{N}_{W}(R \cap T)$. Since $\mathbf{N}_{W}(Q) \subseteq \mathbf{N}_{W}(R)$, we have that $\mathbf{N}_{W}(Q)$ normalizes $R$. Since $\mathbf{N}_{W}(Q) \subseteq W \subseteq T$, it follows that $\mathbf{N}_{W}(Q)$ normalizes $R \cap T$. Hence $\mathbf{N}_{W}(Q) \subseteq \mathbf{N}_{W}(R \cap T)$, and the claim follows.

Now, by induction, we have that $\delta^{R \cap T}$ is irreducible. We claim that this character lies over the irreducible character $\mu=\left(\theta_{p}\right)_{N \cap Q}$. First of all, $N \cap Q \in \operatorname{Syl}_{p}(N)$, because $Q \in \operatorname{Syl}_{p}(W)$ and $N \triangleleft W$. Hence $\mu$ is irreducible by Theorem (2.1.c). Now, since $\gamma$ lies over $\theta$, by the uniqueness in the factorization of $p$-factorable characters, it follows that $\theta_{p}$ lies under $\beta$. Hence, $\mu$ lies under $\beta$, and therefore under $\beta_{Q}=\delta$. Hence, $\mu$ lies under $\delta^{R \cap T}$, as claimed.

Now, observe that $N \cap Q=N \cap R \triangleleft R$. We claim that $R \cap T=I_{R}(\mu)$ (the stabilizer of $\mu$ in $R$ ). If $x \in R \cap T$, we have that $x$ fixes $\theta$. By the uniqueness of the decomposition of $\theta$, we also have that $x$ fixes $\theta_{p}$. Since $x \in R \subseteq \mathbf{N}_{G}(Q \cap N)$, it follows that $x$ fixes $\left(\theta_{p}\right)_{Q \cap N}=\mu$. On the other hand, if $x \in R$ fixes $\mu$, by the uniqueness in Theorem (2.1.c), we have that $x$ fixes $\theta_{p}$. Now, since $\mathbf{N}_{W}(Q) \subseteq$ $\mathbf{N}_{W}(R)$, we have that $\mathbf{N}_{N}(Q) \subseteq \mathbf{N}_{N}(R)$. Since $\theta_{p^{\prime}}$ is $Q$-invariant, by Theorem (4.1) we have that $\theta_{p^{\prime}}$ is $R$-invariant. Hence $x$ fixes $\theta_{p} \theta_{p^{\prime}}=\theta$ and thus $x \in R \cap T$. This proves the claim.

Finally, by the Clifford correspondence (Theorem (6.11) of 4]) we have that

$$
\delta^{R}=\left(\delta^{R \cap T}\right)^{R}
$$

is irreducible, as desired.

The following is Theorem A(f) of the introduction.

(5.2) Corollary. Let $G$ be p-solvable and suppose that $(Q, \delta)$ is a vertex of $\chi \in$ $\operatorname{Irr}(G)$. Let $R$ be the radical closure of $Q$ in $G$. Then $\eta=\delta^{R} \in \operatorname{Irr}(R)$. In particular, $d(\eta)=d(\chi)$. 
Proof. By definition, there is a nucleus $(W, \gamma)$ of $\chi$ such that $Q \in \operatorname{Syl}_{p}(W)$, and $\left(\gamma_{p}\right)_{Q}=\delta$. Since $R$ is the radical closure of $Q$ in $G$, we know that $\mathbf{N}_{G}(Q) \subseteq \mathbf{N}_{G}(R)$. In particular, $\mathbf{N}_{W}(Q) \subseteq \mathbf{N}_{W}(R)$. By Theorem (5.1), we conclude that $\eta=\delta^{R} \in$ $\operatorname{Irr}(R)$. By Lemma (3.1) and Theorem (3.2), we have that $d(\chi)=d(\delta)=d(\eta)$, as desired.

Next, we work towards proving Theorem A(e). We will use the following (which is the essence of the main results in [9]).

(5.3) Theorem. Suppose that $G$ is p-solvable, let $N=\mathbf{O}_{p^{\prime}}(G)$ and let $\chi \in \operatorname{Irr}(G)$. Suppose that $\chi=(\alpha \beta)^{G}$, where $\alpha \in \operatorname{Irr}(U)$ has $p^{\prime}$-degree and $\beta \in \operatorname{Irr}(U)$ is p-special. Suppose that $\chi_{N}$ is homogeneous. If $Q \in \operatorname{Syl}_{p}(U)$, then

$$
\mathbf{C}_{G / N}(Q)=\mathbf{Z}(Q) N / N \text {. }
$$

Proof. This is Theorem (3.5) of [9].

(5.4) Theorem. Let $G$ be $p$-solvable and let $\chi \in \operatorname{Irr}(G)$. Suppose that $\chi=\gamma^{G}$ for some $\gamma \in \operatorname{Irr}(W)$, and suppose that $\gamma=\alpha \beta \in \operatorname{Irr}(W)$, where $\alpha$ has $p^{\prime}$-degree and $\beta$ is p-special. Let $Q \in \operatorname{Syl}_{p}(W)$. Suppose that $Q \subseteq R$ is a p-group such that whenever $R$ normalizes a $p^{\prime}$-subgroup $N$ of $G$, then $\mathbf{C}_{N}(R)=\mathbf{C}_{N}(Q)$. Then there is a defect group $D$ of the p-block of $\chi$ such that $R \subseteq D$ and $\mathbf{C}_{D}(Q) \subseteq Q$.

Proof. We argue by induction on $\left|G: \mathbf{O}_{p^{\prime}}(G)\right|$ and $|G: W|$. Let $N=\mathbf{O}_{p^{\prime}}(G)$. By Theorem (2.1.a), we have that $N \cap W \subseteq \operatorname{ker}(\beta)$. Hence, it easily follows that $\beta$ has a $p$-special extension $\hat{\beta} \in \operatorname{Irr}(W N / N)$. Then

$$
\gamma^{W N}=\left(\alpha \hat{\beta}_{W}\right)^{W N}=\alpha^{W N} \hat{\beta} ;
$$

notice that here $\alpha^{W N} \in \operatorname{Irr}(W N)$ has $p^{\prime}$-degree. Also, $Q \in \operatorname{Syl}_{p}(W N)$. If $W N>$ $W$, then by induction, we are done. So we may assume that $N \subseteq W$. Now, let $\theta \in \operatorname{Irr}(N)$ be an irreducible constituent of $\gamma_{N}$ and let $T$ be the stabilizer of $\theta$ in $G$. Since $N \subseteq \operatorname{ker}(\beta)$, we have that $\theta$ is an irreducible constituent of $\alpha_{N}$. Since $\alpha$ has $p^{\prime}$-degree, we may assume that $\theta$ is $Q$-invariant (since $Q$ permutes the irreducible constituents of $\left.\alpha_{N}\right)$. Now, $R$ normalizes $N$, and by hypothesis, we have that $\mathbf{C}_{N}(R)=\mathbf{C}_{N}(Q)$. By Theorem (4.1), we conclude that $\theta$ is $R$-invariant. Thus $R \subseteq T$. Now, $Q \subseteq T \cap W$, and then $T \cap W$ has $p^{\prime}$-index in $W$. Therefore $\beta_{T \cap W}$ is irreducible and $p$-special by Theorem (2.1.c). Now, let $\eta$ be the Clifford correspondent of $\alpha$ over $\theta$, which has $p^{\prime}$-degree. We have

$$
\left(\eta \beta_{T \cap W}\right)^{W}=\eta^{W} \beta=\alpha \beta
$$

and therefore

$$
\left(\eta \beta_{T \cap W}\right)^{G}=\chi .
$$

In particular, $\left(\beta_{T \cap W} \eta\right)^{T}=\mu$ is the Clifford correspondent of $\chi$ over $\theta$. If $T<G$, by induction, there is a defect group $D$ of the block of $\mu$ satisfying $R \subseteq D$ and $\mathbf{C}_{D}(Q) \subseteq Q$. Now, by Theorem (9.14) of [8],D is a defect group of the block of $\chi$, and we are done in this case. So we may assume that $T=G$, and that the defect groups of the block of $\chi$ are the Sylow $p$-subgroups of $G$ (by Theorem (10.20) of [8]). Now, by Theorem (5.3), we know that

$$
\mathbf{C}_{G / N}(Q)=\mathbf{Z}(Q) N / N .
$$


In particular, $\mathbf{C}_{G}(Q) \subseteq W$. Now, let $Q \subseteq R \subseteq D \in \operatorname{Syl}_{p}(G)$. Then $\mathbf{C}_{D}(Q) \subseteq W$. Since $Q \mathbf{C}_{D}(Q)$ is a $p$-subgroup of $W$ containing $Q \in \operatorname{Syl}_{p}(W)$, we conclude that $\mathbf{C}_{D}(Q) \subseteq Q$, as desired.

This is Theorem $\mathrm{A}(\mathrm{e})$ of the Introduction.

(5.5) Theorem. Let $G$ be $p$-solvable and suppose that $(Q, \delta)$ is a vertex of $\chi$. Let $R$ be the radical closure of $Q$ in $G$. Then there is a defect group $D$ of the p-block of $\chi$ such that $Q \subseteq R \subseteq D$ and

$$
\mathbf{C}_{D}(Q)=\mathbf{Z}(Q) .
$$

Proof. Let $(W, \gamma)$ be a nucleus for $\chi$ with $Q \in \operatorname{Syl}_{p}(W)$. Now, since $\mathbf{N}_{G}(Q) \subseteq$ $\mathbf{N}_{G}(R)$, it follows that if $R$ normalizes any $p^{\prime}$-subgroup $N$ of $G$, then $\mathbf{C}_{N}(Q)=$ $\mathbf{N}_{N}(Q) \subseteq \mathbf{N}_{N}(R)=\mathbf{C}_{N}(R)$. Now, Theorem (5.4) applies.

\section{Lifting Brauer characters}

We need the following.

(6.1) Lemma. Suppose that $G$ is p-solvable, and let $\varphi \in \operatorname{IBr}(G)$.

(a) Suppose that $G=M N$, where $M, N \triangleleft G$. If the irreducible constituents of $\varphi_{N}$ and of $\varphi_{M}$ have $p^{\prime}$-degree, then $\varphi$ has $p^{\prime}$-degree.

(b) There exists a unique maximal normal subgroup $E$ of $G$ such that the irreducible constituents of $\varphi_{E}$ have $p^{\prime}$-degree. Furthermore, if $\varphi_{E}=e \theta$ for some $\theta \in \operatorname{IBr}(E)$, then $\varphi$ has $p^{\prime}$-degree.

Proof. (a) Arguing as in Theorem (2.2), we may easily assume that $G / N$ and $G / M$ are simple groups. If, for instance, $G / N$ is a $p^{\prime}$-group, then $\varphi$ has $p^{\prime}$-degree by Theorem (8.30) of [8]. So we may assume that $G / N \cap M$ is a $p$-group. Now, let $\delta \in \operatorname{IBr}(N \cap M)$ lie under $\varphi$. If $\theta \in \operatorname{IBr}(N)$ lies under $\varphi$ and over $\delta$, we have that $\theta_{N \cap M}=\delta$. Hence, $\delta$ is $N$-invariant. By the same argument, $\delta$ is $M$-invariant. Hence, $\delta$ is $G$-invariant. Also, since $\theta$ has $p^{\prime}$-degree, so does $\delta$. By Green's theorem (Theorem (8.11) of [8]), $\varphi_{N \cap M}=\delta$, and the proof of the first part follows.

(b) It is clear that such a normal subgroup $E$ exists, by part (a). Also, by Theorem (8.30) of [8], we have that $\mathbf{O}_{p^{\prime}}(G / E)=1$. So if $E<G$, we may find a normal subgroup $K$ of $G$ with $K / E$ a $p$-group. By Green's theorem, there is a unique irreducible Brauer character $\tau$ of $K$ which lies over $\theta$. In fact, $\tau$ extends $\theta$, and therefore also has $p^{\prime}$-degree. By uniqueness, we have that $\varphi_{K}=e \tau$. This contradicts the maximality of $E$.

For our convenience, we will use the following characterization of the vertex of an irreducible Brauer character of a $p$-solvable group. If $G$ is $p$-solvable and $\varphi \in \operatorname{IBr}(G)$, by Huppert's theorem (Theorem (10.11) of [8]), we know that there is some $\alpha \in \operatorname{IBr}(U)$ of $p^{\prime}$-degree, such that $\alpha^{G}=\varphi$. In fact, if $\beta \in \operatorname{IBr}(V)$ is some other irreducible Brauer character of $p^{\prime}$-degree, it is a fact that the Sylow $p$ subgroups of $U$ and $V$ are $G$-conjugate. (Apply Theorem B of [5] with $\pi=p^{\prime}$.) This conjugacy class of $p$-subgroups of $G$ uniquely determined by $\varphi$ up to $G$-conjugacy are the vertices of $\varphi$. Recall that we denote by $\operatorname{IBr}(G \mid Q)$ the set of irreducible Brauer characters of $G$ with vertex $Q$.

As a consequence of the definition, notice that if some Brauer character $\mu$ induces $\varphi$, then every vertex of $\mu$ is a vertex of $\varphi$. 
Fix a $p$-subgroup $Q$ of a $p$-solvable group $G$ and suppose that $\varphi \in \operatorname{IBr}(G \mid Q)$. Let $N \triangleleft G$, and let $\theta \in \operatorname{IBr}(N)$ lie under $\varphi$. We say that $\theta$ is $Q$-good for $\varphi$ if the Clifford correspondent of $\varphi$ over $\theta$ has vertex $Q$.

(6.2) Lemma. Suppose that $G$ is p-solvable, let $Q$ be a p-subgroup of $G$ and let $\varphi \in \operatorname{IBr}(G \mid Q)$. Suppose that $N \triangleleft G$. Then there is an irreducible constituent $\theta$ of $\varphi_{N}$ which is Q-good, and any two of them are $\mathbf{N}_{G}(Q)$-conjugate.

Proof. Let $\eta \in \operatorname{IBr}(N)$ be any irreducible constituent of $\varphi_{N}$. Let $I$ be the inertia group of $\eta$ in $G$ and let $\mu \in \operatorname{IBr}(I)$ be the Clifford correspondent of $\varphi$ over $\eta$. Let $P$ be a vertex of $\mu$. Hence, $P$ is a vertex of $\varphi$, and we deduce that $P^{g}=Q$ for some $g \in G$. Let $\theta=\eta^{g}$. Then $T=I^{g}$ is the stabilizer of $\theta$ in $G$, and $\tau=\mu^{g}$, which is the Clifford correspondent of $\varphi$ over $\theta$, has vertex $P^{g}=Q$. Suppose now that $\theta^{x}$ is also $Q$-good for $\varphi$ for some $x \in G$. Since $\tau^{x}$ is the Clifford correspondent of $\varphi$ over $\theta^{x}$, we have that $\tau^{x}$ has vertex $Q$. However, since $\tau$ has vertex $Q$, it easily follows that $\tau^{x}$ has vertex $Q^{x}$. Hence, $Q^{x}=Q^{t^{x}}$ for some $t \in T$. Thus $t^{-1} x \in \mathbf{N}_{G}(Q)$ and $\theta^{t^{-1} x}=\theta^{x}$, as desired.

The next result, which is Theorem $\mathrm{A}(\mathrm{a})$ of the Introduction, proves that the irreducible characters of $G$ with vertex $\left(Q, 1_{Q}\right)$ provide a canonical lifting of the irreducible Brauer characters of a finite $p$-solvable group $G$ with vertex $Q$. M. Isaacs constructed in [3] another (apparently) canonical set of liftings $B_{p^{\prime}}(G) \subseteq \operatorname{Irr}(G)$ of $\operatorname{IBr}(G)$. We do know that for $p$ odd, both liftings coincide. However, at the time of this writing, we do not know what happens in general. Perhaps surprisingly, we need the Isaacs $B_{p^{\prime}}$-lifting in order to prove ours. The only facts that we need to know about $B_{p^{\prime}}$-characters are that normal irreducible constituents of $B_{p^{\prime}}$-characters are $B_{p^{\prime}}$-characters (something that we do not know about our lifting) and that the $B_{p^{\prime}}$-characters of $p^{\prime}$-degree are exactly the $p^{\prime}$-special characters.

(6.3) Theorem. Let $Q$ be a p-subgroup of $G$. Then restriction to p-regular elements defines a natural bijection $\operatorname{Irr}\left(G \mid Q, 1_{Q}\right) \rightarrow \operatorname{IBr}(G \mid Q)$.

Proof. We argue by induction on $|G|$. Let $\chi \in \operatorname{Irr}\left(G \mid Q, 1_{Q}\right)$. First, we prove that $\chi^{0} \in \operatorname{IBr}(G \mid Q)$. By the definition of the vertex $\left(Q, 1_{Q}\right)$, we know that there exists a nucleus $(W, \gamma)$ of $\chi$ such that $Q \in \operatorname{Syl}_{p}(W)$ and $\left(\gamma_{p}\right)_{Q}=1_{Q}$. Now, by definition of the nucleus, we know that there exists $(N, \theta)$, a maximal $p$-factorable normal pair under $\chi$, such that $N \subseteq W \subseteq T$, where $T=I_{G}(\theta), \theta$ lies under $\gamma$, and $(W, \gamma)$ is a nucleus for the Clifford correspondent $\psi$ of $\chi$ over $\theta$. Thus $\psi \in \operatorname{Irr}\left(T \mid Q, 1_{Q}\right)$. Now, since $\left(\gamma_{p}\right)_{Q}=1_{Q}$, by Theorem (2.1.c), we have that $\gamma_{p}=1_{W}$, and we deduce that $\gamma$ is $p^{\prime}$-special. Since $\theta$ is under $\gamma$, we deduce that $\theta$ is $p^{\prime}$-special. By Theorem (2.1.e), we have that $\varphi=\theta^{0} \in \operatorname{IBr}(N)$, and by the uniqueness in that result, we have that $T$ is also the stabilizer of $\varphi$ in $G$. If $T=G$, then $\theta=\chi, Q \in \operatorname{Syl}_{p}(G)$ and $\chi^{0}=\varphi \in \operatorname{IBr}(G)$. Since $\varphi$ has $p^{\prime}$-degree, we also have that $Q$ is a vertex of $\varphi$ in this case. So we may assume that $T<G$. By induction, $\psi^{0} \in \operatorname{IBr}(T \mid Q)$. Also, $\psi^{0}$ lies over $\theta^{0}=\varphi$, and by the Clifford correspondence for Brauer characters, we have that

$$
\chi^{0}=\left(\psi^{G}\right)^{0}=\left(\psi^{0}\right)^{G} \in \operatorname{IBr}(G \mid Q) .
$$

Write $\sigma=\chi^{0} \in \operatorname{IBr}(G)$. With the previous notation, we claim that $N$ is the maximal normal subgroup of $G$ such that the irreducible constituents of $\sigma_{N}$ have 
$p^{\prime}$-degree. Suppose that $E$ is such a normal subgroup. Since the irreducible constituents of $\sigma_{N}$ (which are $G$-conjugates of $\varphi$ ) have $p^{\prime}$-degree, we have that $N \subseteq E$. Let $\tau \in \operatorname{IBr}(E)$ lie under $\sigma$ and over $\varphi$. By Theorem (2.1.e), let $\kappa \in \operatorname{Irr}(E)$ be $p^{\prime}$-special such that $\kappa^{0}=\tau$. By Theorem (2.1.f), we have that $\kappa$ lies over $\theta$. Since $(N, \theta)$ is maximal $p$-factorable, we have that $N=E$, as claimed.

Now, we prove that our map is one-to-one. Suppose that $\chi^{0}=\eta^{0}=\sigma$ for some $\eta \in \operatorname{Irr}\left(G \mid Q, 1_{Q}\right)$. We want to prove that $\chi=\eta$. As before, we may find a maximal $p$-factorable pair $(M, \nu)$ for $\eta$ such that the Clifford correspondent $\xi$ of $\eta$ over $\nu$ lies in $\operatorname{Irr}\left(I \mid Q, 1_{Q}\right)$, where $I$ is the inertia group of $\nu$ in $G$. Now, by the previous paragraph, we know that $N$ is the maximal normal subgroup such that the irreducible constituents of $\sigma_{N}$ have $p^{\prime}$-degree. By the same argument applied to $\eta$, we deduce that $N=M$, and that $\theta^{0}$ and $\nu^{0}$ are $G$-conjugate. Hence, by Theorem (2.1.e), we have that $\theta$ and $\nu$ are $G$-conjugate. If $T=G$, then $N=$ $M=G, \eta=\nu=\theta=\chi$, and in this case, we are done. So we may assume that $T$ is proper in $G$. By induction, we have that $\psi^{0}$ and $\xi^{0}$ have vertex $Q$. Since both are Clifford correspondents of $\sigma$ over $N$, by Lemma (6.2), we deduce that $\theta^{0}$ and $\nu^{0}$ (and therefore, $\theta$ and $\nu$ ) are $\mathbf{N}_{G}(Q)$-conjugate. Suppose that $\nu^{n}=\theta$ for $n \in \mathbf{N}_{G}(Q)$. Now, $I^{n}=T$, and $\xi^{n} \in \operatorname{Irr}\left(T \mid Q, 1_{Q}\right)$. Now, $\psi^{0}$ and $\left(\xi^{n}\right)^{0}$ are the Clifford correspondent of $\sigma$ over $\theta$. By uniqueness, $\psi^{0}=\left(\xi^{n}\right)^{0}$, and by induction we have that $\psi=\xi^{n}$. Hence

$$
\chi=\psi^{G}=\left(\xi^{n}\right)^{G}=\eta
$$

and the injectivity is proven.

Finally, we prove that our map is surjective. Suppose that $\varphi \in \operatorname{IBr}(G \mid Q)$. By Lemma (6.1), let $N$ be maximal such that the irreducible constituents of $\varphi_{N}$ have $p^{\prime}$-degree. By Lemma (6.2), let $\theta \in \operatorname{IBr}(N)$ be an irreducible constituent of $\varphi_{N}$ such that, if $T$ is the stabilizer of $\theta$ in $G$ and $\mu \in \operatorname{IBr}(T \mid \theta)$ is the Clifford correspondent of $\varphi$ over $\theta$, then $\mu$ has vertex $Q$. If $T=G$, by Lemma (6.1) we have that $\varphi$ has $p^{\prime}$-degree. In this case $Q \in \operatorname{Syl}_{p}(G)$. By Theorem (2.1.e), there is some $p^{\prime}$-special character $\psi$ of $G$ such that $\psi^{0}=\varphi$. It is clear in this case that $\psi \in \operatorname{Irr}\left(G \mid Q, 1_{Q}\right)$. So we may assume that $T$ is proper in $G$. Now, let $\chi \in \mathrm{B}_{p^{\prime}}(G)$ be an Isaacs lifting for $\varphi$. The irreducible constituents of $\chi_{N}$ are in $\mathrm{B}_{p^{\prime}}(N)$, so they lift irreducible characters of $N$. Hence, there is some $\nu \in \mathrm{B}_{p^{\prime}}(N)$ such that $\nu^{0}=\theta$. Since $\nu$ has $p^{\prime}$-degree, $\nu$ is $p^{\prime}$-special by Lemma (5.4) of [3]. Also, we know that $T=I_{G}(\nu)$ by uniqueness. We claim that $(N, \nu)$ is a maximal $p$-factorable normal pair below $\chi$. Suppose that $N \subseteq M \triangleleft G$ is such that the irreducible constituents of $\chi_{M}$ are $p$ factorable. Let $\eta \in \operatorname{Irr}(M)$ be one of them over $\nu$. Since $\eta \in \mathrm{B}_{p^{\prime}}(M)$ is $p$-factorable, we have that $\eta$ is $p^{\prime}$-special by Lemma (5.4) of [3]. Now $\eta^{0}$ lies under $\varphi$ and has $p^{\prime}$-degree. By the maximality of $N$, we conclude that $N=M$. Thus $(N, \nu)$ is a maximal normal pair, as desired. By induction, there is some $\xi \in \operatorname{Irr}\left(T \mid Q, 1_{Q}\right)$ such that $\xi^{0}=\mu$. By definition, there is some nucleus $(W, \gamma)$ for $\xi$ such that $\gamma$ is $p^{\prime}$-special and $Q \in \operatorname{Syl}_{p}(W)$. Suppose that $(M, \rho)$ is a maximal normal $p$-factorable pair for $\xi$. By the second paragraph, we know that $\rho$ is $p^{\prime}$-special and that $M$ is the maximal normal subgroup of $T$ such that the irreducible constituents of $\mu_{M}$ have $p^{\prime}$-degree. Since $\mu_{N}=e \theta$ and $\theta$ has $p^{\prime}$-degree, we deduce that $N \subseteq M$ and that $\rho^{0}$ lies over $\theta$. By Theorem (2.1.f), we deduce that $\rho$ lies over $\theta$. Hence, $\xi$ lies over $\nu$. Therefore, we conclude that $(W, \gamma)$ is a nucleus of $\xi^{G}$. Therefore, $\xi^{G} \in \operatorname{Irr}\left(G \mid Q, 1_{Q}\right)$ lifts $\varphi$, and the proof of the theorem is complete. 


\section{REFERENCES}

1. L. Barker, Defects of irreducible characters of $p$-soluble groups, J. Algebra 202 (1998), 178184. MR 99b:20014

2. D. Gajendragadkar, A characteristic class of characters of finite $\pi$-separable groups, J. Algebra 59 (1979), 237-259. MR 82b:20012

3. I. M. Isaacs, Characters of $\pi$-separable groups, J. Algebra 86 (1984), 98-128. MR 85h:20012

4. I. M. Isaacs, Character Theory of Finite Groups, Dover, New York, 1994. MR 57:417] (1st ed.)

5. I. M. Isaacs, G. Navarro, Weights and vertices for characters of $\pi$-separable groups, J. Algebra 177 (1995), 339-366. MR 97a:20006

6. I. M. Isaacs, G. Navarro, Characters of $p^{\prime}$-degree of $p$-solvable groups, to appear in J. Algebra.

7. R. Knörr, On the vertices of irreducible modules, Annals of Mathematics 110 (1979), 487-499. MR 81f:20013

8. G. Navarro, Characters and Blocks of Finite Groups, London Math. Soc. Lecture Note Series 250, Cambridge University Press, 1998. MR 2000a:20018

9. G. Navarro, Induction of characters and $p$-subgroups, to appear in J. Algebra.

10. G. R. Robinson, Local structure, vertices and Alperin's conjecture, Proc. London Math. Soc. (3) 72, (1996), 312-330. MR 97c:20015

11. G. R. Robinson, Dade's projective conjecture for $p$-solvable groups. J. Algebra 229 (2000), 234-248. MR 2001h:20013

12. T. R. Wolf, Variations on McKay's character degree conjecture, J. Algebra 135 (1990), 123138. MR 91h:20023

Departament d’Àlgebra, Facultat de Matemàtiques, Universitat de València, 46100 Burjassot. València, Spain

E-mail address: gabriel@uv.es 